\title{
Highly-efficient Cas9-mediated transcriptional programming
}

Alejandro Chavez $z^{1,2,3,{ }^{*},}$ Jonathan Scheiman ${ }^{1,3, *}$, Suhani Vora ${ }^{1,3,4,}$, , Benjamin W. Pruitt ${ }^{1}$, Marcelle Tuttle ${ }^{1}$, Eswar Iyer ${ }^{1,3}$, Samira Kiani, ${ }^{4,5}$, Christopher D. Guzman ${ }^{1}$, Daniel J. Wiegand ${ }^{1}$, Dimtry Ter-Ovanesyan ${ }^{1,3}$, Jonathan L. Braff ${ }^{1}$, Noah Davidsohn ${ }^{1,3}$, Ron Weiss ${ }^{4,5}$, John Aach ${ }^{3}$, James J. Collins ${ }^{1,6,7}$, George M. Church ${ }^{1,3}$

\author{
${ }^{1}$ Wyss Institute for Biologically Inspired Engineering, Harvard University, Cambridge, MA 02138, USA. \\ 2Department of Pathology, Massachusetts General Hospital, Boston, MA 02114, USA. \\ ${ }^{3}$ Department of Genetics, Harvard Medical School, Boston, MA 02115, USA. \\ ${ }^{4}$ Department of Biological Engineering, Massachusetts Institute of Technology, Cambridge, MA 02139, USA. \\ ${ }^{5}$ Synthetic Biology Center, Massachusetts Institute of Technology, Cambridge, MA 02139 \\ ${ }^{6}$ Department of Biomedical Engineering and Center of Synthetic Biology, Boston University, Boston, MA 02215, USA \\ ${ }^{7}$ Howard Hughes Medical Institute, Chevy Chase, MD 20815, USA \\ *These authors contributed equally \\ Correspondence should be addressed to G.M.C. (gchurch@genetics.med.harvard.edu)
}

\section{ABSTRACT}

The RNA-guided bacterial nuclease Cas9 can be reengineered as a programmable transcription factor by a series of changes to the Cas9 protein in addition to the fusion of a transcriptional activation domain $(A D)^{1-5}$. However, the modest levels of gene activation achieved by current Cas 9 activators have limited their potential applications. Here we describe the development of an improved transcriptional regulator through the rational design of a tripartite activator, VP64-p65-Rta (VPR), fused to Cas9. We demonstrate its utility in activating expression of endogenous coding and non-coding genes, targeting several genes simultaneously and stimulating neuronal differentiation of induced pluripotent stem cells (iPSCs).

\section{RESULTS}

Cas9 is an RNA-guided endonuclease that is directed to a specific DNA sequence through complementarity between the associated guide RNA (gRNA) and its target locus ${ }^{6-9}$. Cas9 can be directed to nearly any arbitrary sequence with a gRNA, requiring only a short protospacer adjacent motif (PAM) site proximal to the target ${ }^{10-14}$. Through mutational analysis, variants of Cas9 have been generated that lack endonucleolytic activity but retain the capacity to interact with DNA $1,9,15,16$. These nuclease-null or dCas9 variants have been subsequently functionalized with effector domains such as transcriptional activation domains (ADs), enabling Cas9 to serve as a tool for cellular programming at the transcriptional level ${ }^{1-5}$. The ability to programmably induce expression of a specific target within its native chromosomal context would provide a transformative tool for myriad applications, including the development of therapeutic interventions, genetic screening, activation of endogenous and synthetic genetic circuits, and the induction of cellular differentiation ${ }^{17-20}$.
To design an enhanced programmable activator, we screened a library of transcription factor ADs and members of the Mediator and RNA polymerase II complexes for potential use as transcriptional effectors ${ }^{21}$. More than 20 individual candidates were fused to the C-terminus of Streptococcus pyogenes (SP)-dCas9. These putative dCas9-activator constructs were transfected into mammalian cells and their potency assessed by quantifying fluorescence from a transcriptional reporter by flow cytometry ${ }^{1}$ (Supplemental Fig. 1). Of the hybrid proteins tested, three - dCas9-VP64, dCas9-p65 and dCas9-Rta showed meaningful reporter induction ${ }^{2,22}$. Nonetheless, neither the p65 nor the Rta hybrid were stronger activators than the commonly used dCas9-VP64 protein (Supplemental Fig. 2).

In natural systems, transcriptional initiation occurs through the coordinated recruitment of the necessary machinery by a group of locally concentrated transcription factor $\mathrm{ADs}^{23}$. Hence, we hypothesized that joining multiple activation domains to a single dCas9 molecule would result in an increase in 
A

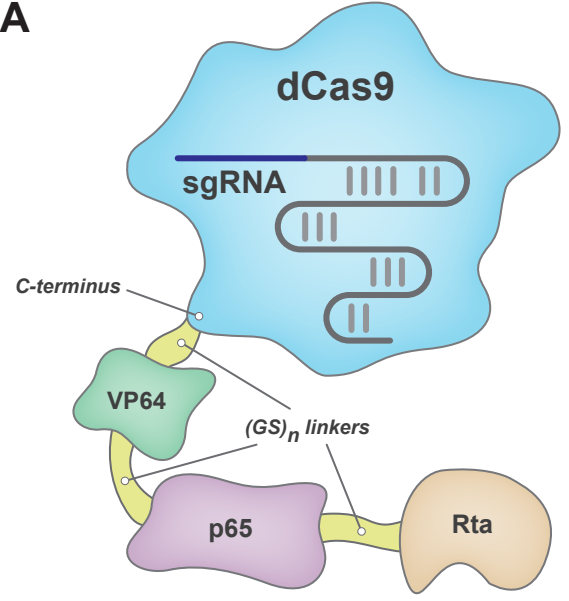

B

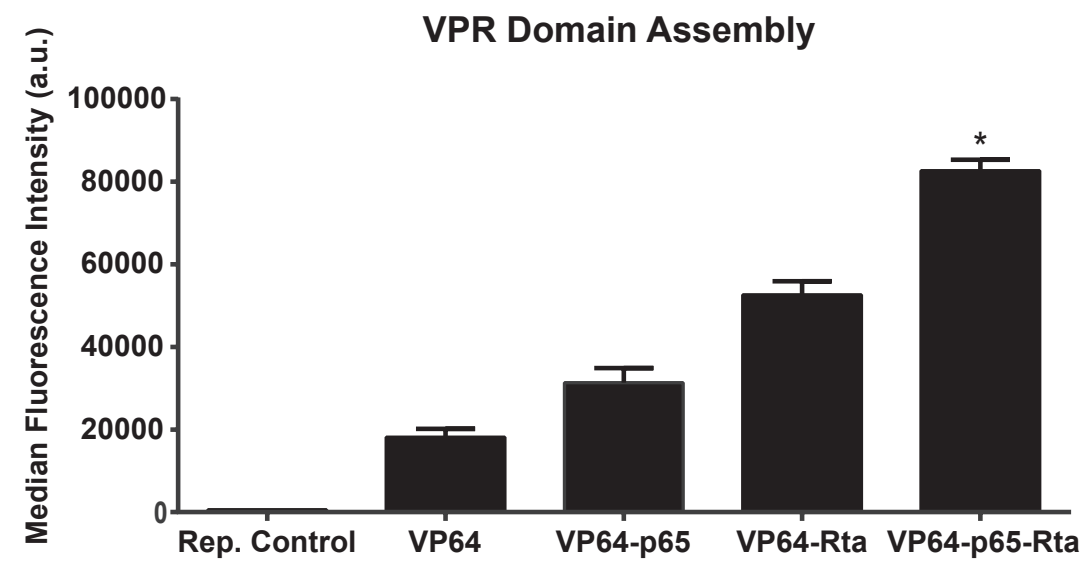

Figure 1 | Activation is improved by serial fusion of activation domains to dCas9. (A) Transcriptional activation via Cas9 was performed by fusing three activation domains, VP64, p65, and Rta, to the C-terminus of a nuclease-null dCas9 protein. (B) Serial activation domain assemblies fused to dCas9 were tested against a fluorescent reporter assay. Data represent median fluorescence \pm s.e.m. $n=5$ biological replicates, performed in a single experiment. ( ${ }^{*}$ denotes significance of dCas9-VP64-p65-Rta over all constructs including Reporter Control P $=<0.0001$, dCas9-VP64 P $=<0.0001$, dCas9VP64-p65 P = <0.0001, and dCas9-VP64-Rta $P=<0.0001)$.

transcriptional activation by mimicking the natural cooperative recruitment process. Taking dCas9-VP64 as a starting scaffold, we introduced an additional nuclear localization signal to ensure efficient targeting. We subsequently extended the C-terminal fusion with the addition of either the p65 or Rta AD. As predicted, when p65 or Rta was joined to dCas9-VP64, we observed an increase in transcriptional output. Further improvement was observed when both p65 and Rta were fused in tandem to VP64, generating a hybrid VP64-p65-Rta tripartite activator (hereon referred to as VPR) (Fig. 1).

To begin characterizing VPR, we confirmed the importance of each of its constituent parts (VP64, p65, and Rta) by replacing each member with mCherry and measuring the resulting protein's activity by reporter assay. All fusions containing mCherry showed a decrease in activity relative to VPR but exhibited higher levels of activity with respect to the VP64 activation domain alone (Supplemental Fig. 3), demonstrating the essentiality of all members of the VPR complex. While the VPR fusion showed a clear improvement in transcriptional activation, the optimal ordering of the individual ADs remained unknown. To test the role of domain order, we designed a set of dCas9-VPR constructs in which we shuffled the positions of VP64, p65, and Rta to generate all possible non-repeating arrangements. Evaluation of the VPR permutations confirmed that the original VP64-p65-Rta order was indeed the optimal configuration (Supplemental Fig. 4).

Given the activation efficiency of our SP-dCas9-
VPR fusion, we investigated whether the VPR construct would exhibit similar potency when fused to other DNA-binding scaffolds. Fusion of VPR to a nucleasenull Streptococcus thermophilus (ST1)-dCas9, a designer transcription activator like effector (TALE), or a zinc-finger protein allowed for a respective $\sim 6 x$, $\sim 4 \mathrm{x}$, and $\sim 2 \mathrm{x}$ increase in activation relative to VP64, as determined by reporter assays (Supplemental Fig. $5)^{19,24}$.

Having performed initial characterization of our SP-dCas9-VPR fusion, we sought to assess its ability to activate endogenous coding and non-coding targets relative to VP64. To this end, we constructed 3-4 gRNAs against a set of factors related to cellular reprogramming, development, and gene therapy. When compared to the dCas9-VP64 activator, dCas9VPR showed 22-to-320 fold improved activation of endogenous targets. Induced genes include the longnoncoding RNA (IncRNA) MIAT, the largest proteincoding gene in the human genome, TTN, several transcription factors, NEUROD1, ASCL1, RHOXF2, and a structural protein, $A C T C 1$ (Fig. 2A).

Cas 9 enables multiplexed activation through the simple introduction of a collection of guide RNAs against a desired set of genes. To determine the efficiency of multi-gene targeting, we performed a pooled activation experiment simultaneously inducing four of our initially characterized genes MIAT, NEUROD1, ASCL1, and RHOXF2. VPR allowed for robust multi-locus activation, exhibiting several-fold higher expression levels than VP64 across the panel of genes (Fig. 2B).

The ability to regulate gene expression levels 
A
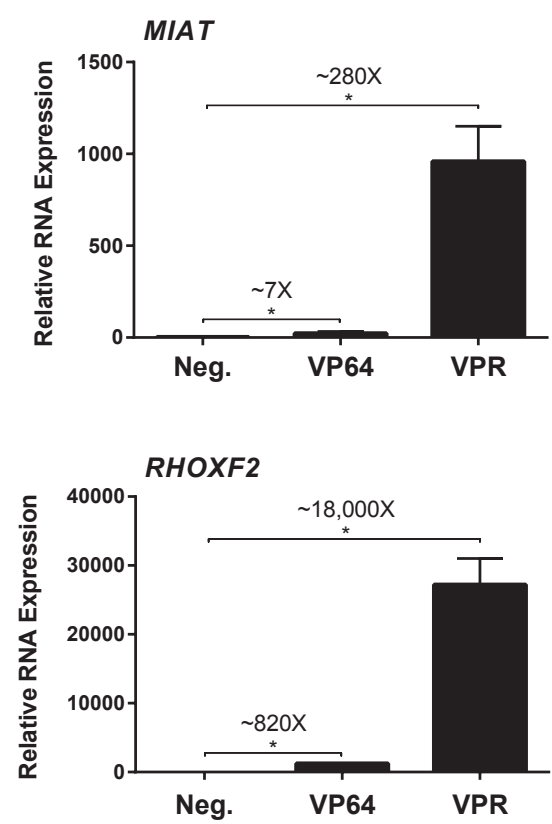

B

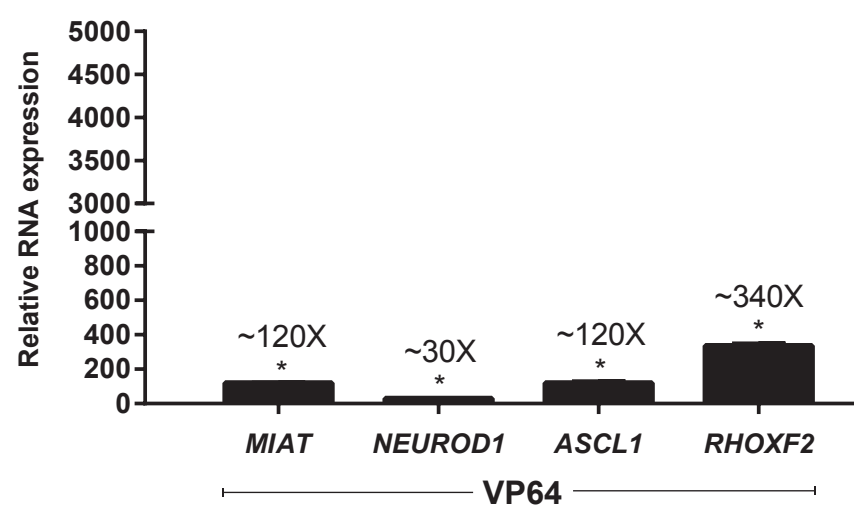

\section{Activation of Endogenous Genes}
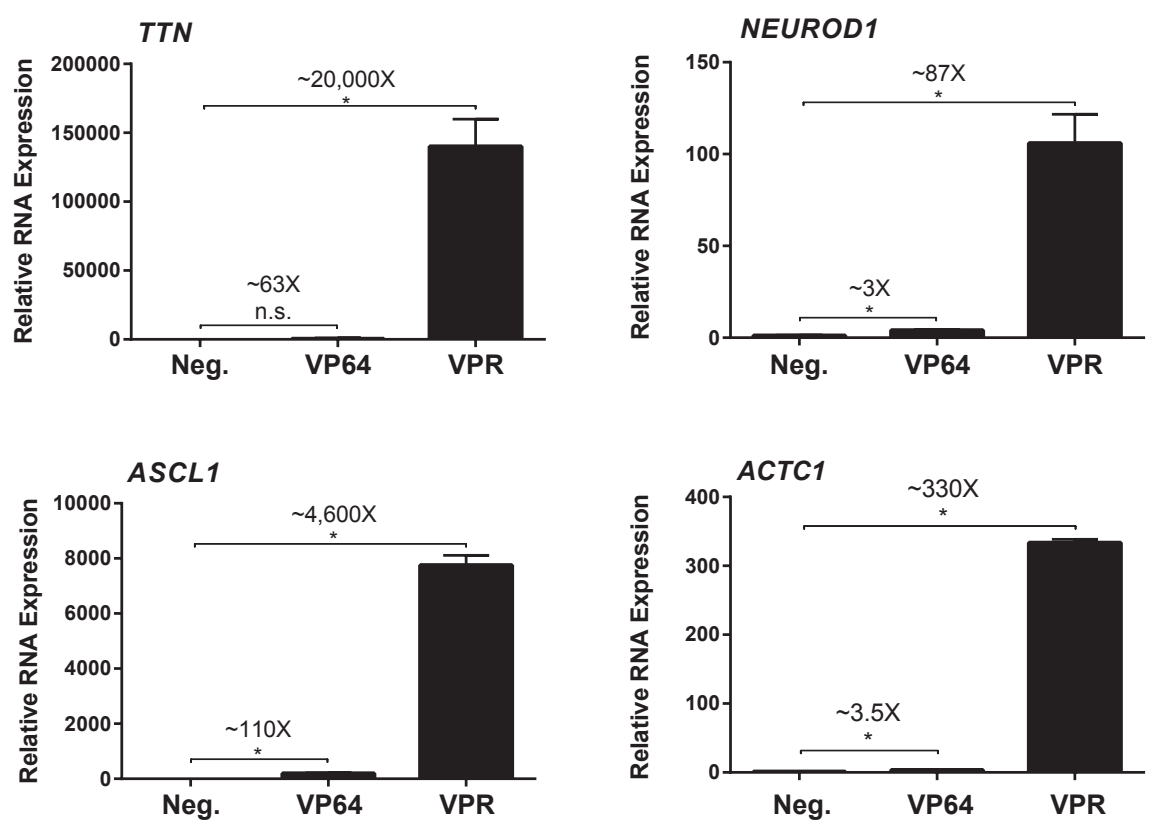

\section{Multiplexed Activation of Endogenous Genes}

Figure 2 | VPR represents a robust tool for gene activation. (A) Target gene expression, measured by qRT-PCR and normalized to Beta-actin mRNA levels, in HEK293T cells simultaneously transfected with 3-4 gRNAs targeting the indicated genes along with the labeled dCas9-activator construct (gRNAs used for activation are listed in the Supplemental Data). Negative controls were transfected with indicated gRNAs alone. Data are shown as the mean \pm s.e.m $(n=3$ biological replicates, performed in a single experiment). Difference in activation between VPR and VP64 was 40-fold, 320-fold, 26-fold, 22-fold, 40-fold, and 94-fold, for MIAT, TTN, NEUROD1, RHOXF2, ASCL1, and ACTC1, respectively. P values determined by two-tailed t-test ( ${ }^{*} \mathrm{dCas} 9-\mathrm{VP} 64$ or dCas9-VPR versus gRNA-only control, respectively, MIAT $\mathrm{P}=0.0277,0.001, T T N$ $\mathrm{P}=$ n.s., 0.0003, NEUROD1 $\mathrm{P}=0.0009,0.0003$, ASCL1 $\mathrm{P}=<0.0001,<0.0001$, RHOXF2 $\mathrm{P}=<0.0001,0.0002$, ACTC1 $\mathrm{P}=0.0002,<0.0001$. n.s. $=$ not significant. Comparison of dCas9-VP64 vs. dCas9-VPR, for all genes, is significant $P=$ $<0.0011)$. (B) Performed as in panel A except 3-4 gRNAs against each of the four endogenous genes, MIAT, NEUROD1, ASCL1, and RHOXF2, were transfected in unison. Data are shown as the mean \pm s.e.m $(\mathrm{n}=3$ biological replicates, performed in a single experiment). P values determined by two-tailed t-test ( ${ }^{*}$ multiplexed dCas9-VP64 or dCas9-VPR vs. mock control sample for each gene respectively, MIAT $\mathrm{P}=1.26 \times 10^{-5}, \mathrm{P}=1.26 \times 10^{-4}$, NEUROD1 $\mathrm{P}=7.28 \times 10^{-5}, \mathrm{P}=4.72 \times 10^{-5}$, ASCL1 P $=5.82 \times 10^{-4}, \mathrm{P}=1.50 \times 10^{-5}$, RHOXF2 P $=1.57 \times 10^{-5}, \mathrm{P}=1.84 \times 10^{-5}$. Comparison of dCas9-VP64 vs. dCas9$\mathrm{VPR}$, for all genes, is significant $\mathrm{P}=<0.0022$ ). 
through transcriptional activation provides a powerful means to reprogram cellular identity for regenerative medicine and basic research purposes. Previous work has shown that the ectopic expression of several cDNAs enables cellular reprogramming of terminally differentiated cells to a pluripotent state, and can similarly induce differentiation of stem cells into multiple cell types ${ }^{25}$. While such studies typically require multiple factors, it was recently shown that exogenous expression of single transcription factors, Neurogenin2 (NGN2) or Neurogenic differentiation factor 1 (NEUROD1), is sufficient to induce differentiation of human iPS cells into induced neurons (iNeurons) ${ }^{26,27}$. Our group had previously attempted to recapitulate this same differentiation paradigm using dCas9-VP64 based activators and observed minimal differentiation activity (data not shown). We hypothesized this was due to insufficient activation and therefore postulated that VPR might overcome this barrier and enable differentiation of iPS cells to iNeurons by more potently activating either NGN2 or NEUROD1.

Stable PGP1 iPS doxycycline-inducible dCas9VP64 and dCas9-VPR cell lines were generated and transduced with lentiviral vectors containing a mixed pool of 30 gRNAs directed against either NGN2 or NEUROD1. To determine differentiation efficiency, gRNA containing dCas9-AD iPS cell lines were cultured in the presence of doxycycline for four days and monitored for phenotypic changes (Supplemental Fig. 6 \& 7). We observed that VPR, in contrast to VP64, enabled rapid and robust differentiation of iPS cells into a neuronal phenotype consistent with previously published reports. Additionally, these cells stained positively for the neuronal markers Beta III tubulin and neurofilament 200 (Fig. 3A and Supplemental Fig. 8A, respectively ${ }^{28}$. Quantification of Beta III tubulin staining
A

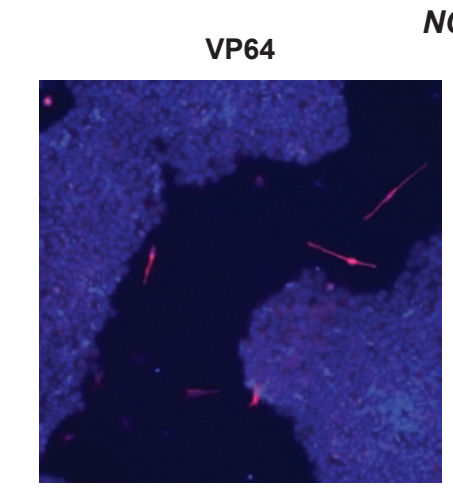

NGN2

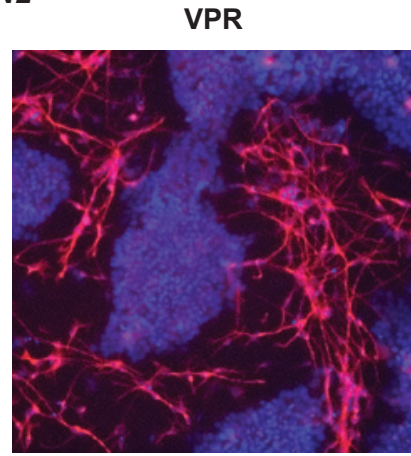

B

iNeuron Efficiency

NGN2

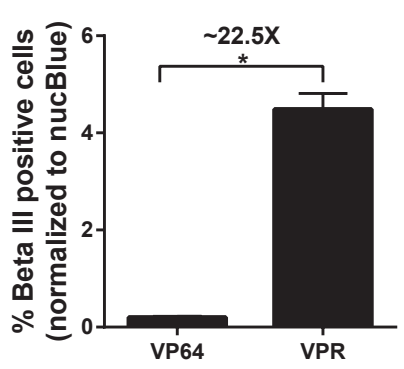

NEUROD1

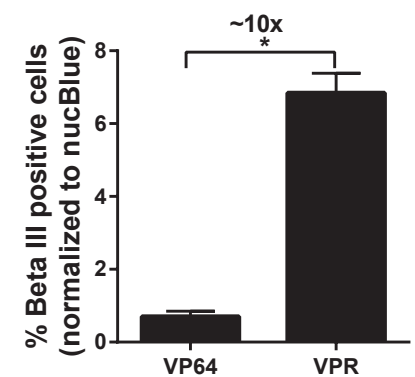

Immunofluorescence

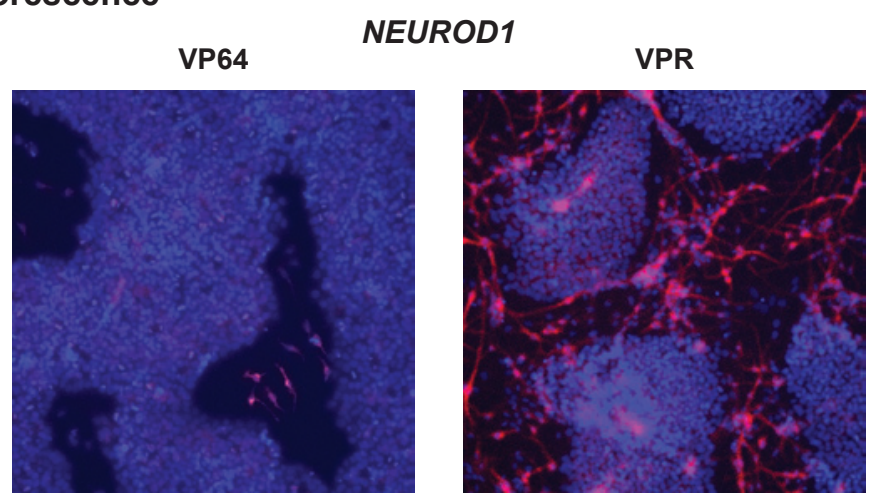

C

mRNA Expression

NGN2

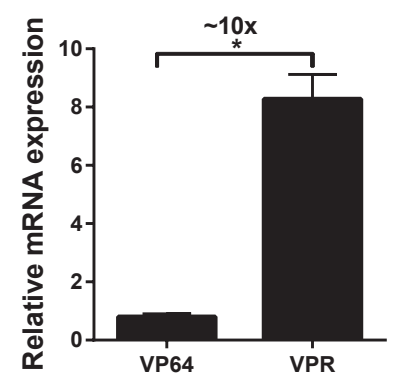

NEUROD1

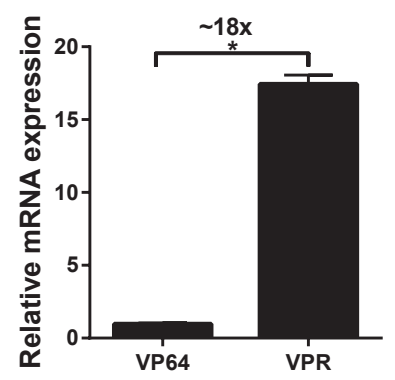

Figure 3 | VPR is essential for efficient dCas9-mediated iPSC neuronal differentiation. (A) Immunofluorescence images for NucBlue (blue, total cells) and Beta III tubulin (red, iNeurons). Images were taken 4 days after doxycycline induction and are representative of biological triplicates. (B) Quantification and comparison of iNeurons generated by either dCas9-VP64 or dCas9-VPR in the presence of doxycycline. Percentages of iNeurons were determined by normalizing Beta III positive cells to total (NucBlue) cells. Data are shown as the mean \pm s.e.m. ( $n=3$ biological replicates, performed in a single experiment, with each replicate being an average of 24 separate images). $\mathrm{P}$ values determined by two-tailed t-test ( ${ }^{*}$ doxycycline induced dCas9-VPR versus doxycycline induced dCas9-VP64. P $=0.0002$ and 0.0004 for NGN2 and NEUROD1, respectively) (C) qRT-PCR analysis for mRNA expression levels of NGN2 and NEUROD1 in dCas9-AD iPS cell lines. Data is normalized to dCas9-VP64 cells and shown as the mean \pm s.e.m. $(n=2$ biological replicates, performed in a single experiment). $\mathrm{P}$ values determined by two-tailed t-test ( ${ }^{*}$ doxycycline induced dCas9-VPR versus doxycycline induced dCas9-VP64. $\mathrm{P}=0.0013$ and $<0.0001$ for NGN2 and NEUROD1, respectively). 
revealed that dCas9-VPR cell lines showed either $\sim 22.5 \mathrm{x}$ or $\sim 10 \mathrm{x}$ improvement in the amount of iNeurons observed through activation of NGN2 or NEUROD1, respectively (Fig. 3B). Similar results were observed with neurofilament 200 staining (Supplemental Fig. $8 B)$. Analysis by qRT-PCR four days after doxycyclineinduction revealed a $\sim 10$-fold and $\sim 18$-fold increase in mRNA expression levels for NGN2 and NEUROD1, respectively, within dCas9-VPR cells over their dCas9VP64 counterparts (Fig. 3C).

\section{DISCUSSION}

Here we introduce a highly-efficient Cas9 transcriptional activator. We demonstrate its versatility in both single and multi-target contexts along with its suitability for cellular reprogramming.

To identify more potent transcriptional effectors, we screened over 20 candidates previously shown to be involved in transcription initiation. While the majority of domains failed to show appreciable activity, the fusions with VP64 (derived from a herpes virus transcription factor), p65 (a member of the NFKB signaling pathway), and Rta (an activator of EpsteinBarr virus genes), showed appreciable activity when fused to dCas9. Previous biochemical analysis of these ADs has determined that each interacts with several members of the transcriptional and chromatin remodeling machinery - VP64 with MED17 and MED25, p65 with MED17, CBP, and P300, and Rta with TBP and TFIIB ${ }^{21,29,30}$. We speculate that the ability of VPR to simultaneously interact with multiple proteins involved in transcription explains the improvement in gene activation that we observe. Furthermore, given the abundance of factors with characterized roles in localized transcriptional initiation, we anticipate that further screening and combinatorial assembly of additional effector peptides will prove fruitful.

While VPR is a single tripartite activator construct fused to dCas9, recent evidence has demonstrated that multimeric recruitment of even the modestly effective VP64 AD can lead to abundant increases in transcriptional activation ${ }^{31}$. We expect that our more potent VPR fusion will be amenable to such multimerization strategies and thereby further enhance our ability to activate endogenous gene targets.

In this study, we focused primarily on identifying and engineering transcriptional ADs. Future designs, however, may directly employ a variety of regulatory proteins that alter chromatin state at the nucleotide, histone, and chromosomal levels ${ }^{32}$. This may prove useful for gaining access to target loci in heterochromatinized regions of the genome.
Interestingly, preliminary evidence suggests that chromatin state may present a less significant impediment to programmable activation than anticipated; we were able to robustly activate RHOXF2, despite it being located in locus repressed by CpG methylation $^{33}$. Therefore, VPR appears to be able to overcome some recalcitrant chromosomal states.

Previous work pioneered Cas9-mediated cellular differentiation, yet to our knowledge we are the first to use Cas9 effectors to successfully generate a targeted cell population (iNeurons) ${ }^{34}$. Furthermore, we demonstrate that effective differentiation is contingent upon the use of the more potent VPR activator. While the amount of iNeurons we observe upon dCas9-VPR mediated differentiation is less than $10 \%$ of the total cell population, it should be noted that the true proportion of differentiated cells is likely higher. Undifferentiated iPS cells continue to divide rapidly during our 4-day induction period while iNeurons are post-mitotic and thus remain under-represented at our analysis endpoint ${ }^{26,27}$.

Another promising facet of our work is the ability to upregulate both endogenous coding and non-coding genes, paving the way for large-scale genomic screens free from constraints such as gene size or availability of previously constructed cDNA libraries ${ }^{35}$. This is particularly relevant in the context of IncRNAs, for which no large-scale expression library exists. Furthermore, by allowing for robust expression of genes from their native loci, we expect to maintain isoform heterogeneity within the particular cell type being tested, enabling a more thorough understanding of gene activity ${ }^{36}$. Finally, the ability to potently upregulate several target genes in unison will prove useful for future applications in directed cellular differentiation, genetic screening, gene network analysis and synthetic circuit engineering.

\section{MATERIALS AND METHODS}

Vectors used and designed. Activation domains were cloned using a combination of Gibson and Gateway assembly or Golden Gate assembly methods. For experiments involving multiple activation domains, ADs were separated by short glycine-serine linkers. All SPdCas9 plasmids were based on Cas9m4-VP64 (Addgene \#47319), ST1-dCas9 plasmids were based on M-ST1nVP64 (Addgene \#48675). Sequences for gRNAs are listed in the Supplemental Information. gRNAs for endogenous gene activation were selected to bind anywhere between 1 and $1000 \mathrm{bp}$ upstream of the transcriptional start site. gRNAs for iPSC differentiation to iNeurons, targeting NGN2 and ND1, were selected to bind anywhere between 1 and 2000 base pairs upstream of the transcriptional start site. 
All gRNAs were expressed from either cloned plasmids (Addgene \#41817) or integrated into the genome through lentiviral delivery (plasmid SB700). gRNA sequences are listed within the Supplemental Data. Reporter targeting gRNAs were previously described (Addgene \#48671 and \#48672).

Cell culture and transfections. HEK 293T cells were maintained in Dulbecco's Modified Eagle's Medium (Invitrogen) with high glucose supplemented with 10\% FBS (Invitrogen) and penicillin/streptomycin (Invitrogen). Cells were maintained at $37^{\circ} \mathrm{C}$ and $5 \% \mathrm{CO}_{2}$ in a humidified incubator. Cells were transfected in 24-well plates seeded with 50,000 cells per well, 200ng of dCas9 activator, 10ng of gRNA plasmid and 60ng of reporter plasmid (when required) were delivered to each well using 2.5 ul of Lipofectamine 2000. For multiplex activation 10ng of gRNA plasmid was used against each target (for example if three guide RNAs were used against a target, a total of $30 \mathrm{ng}$ of guide RNA plasmid was added). Cells were grown an additional 36-48 hours before being assayed using fluorescence microscopy, flow cytometry or lysed for RNA purification and quantification.

Fluorescence Reporter Assay SP-Cas9 reporter assays were performed by targeting all dCas9-ADs with a single guide to a minimal CMV promoter, driving expression of a fluorescent reporter. Addgene plasmid \#47320 was used to screen for novel ADs (Supplemental Figures 1B and 2) or was altered to contain a sfGFP reporter gene instead of tdTomato for Figure 1B, Supplemental Figures 3 and 4. In addition, to control for transfection efficiency in Figure 1B, Supplemental Figures 3 and 4, an EBFP2 expressing control plasmid was co-transfected at $25 \mathrm{ng}$ per well (EBFP2 plasmid was not co-transformed in Supplemental Figure 2). To remove untransfected cells from the analysis, sfGFP fluorescence was only analyzed in cells with $>10^{3}$ EBFP2 expression (as determined by flow cytometry). For fusion of VPR to other programmable transcription factors (ST1dCas9, TALE, and zinc-finger protein) no EBFP2 plasmid was co-transfected. ST1-Cas9 reporter assays were performed using the previously described tdTomato reporter with an appropriate PAM inserted upstream of the Tdtomato coding region. (Addgene \#48678) ${ }^{24}$.

qRT-PCR analysis. RNA was extracted from cells using the RNeasy PLUS mini kit (Qiagen) according to manufacturer's protocol. 500ng of RNA was used with the iScript cDNA synthesis Kit (BioRad), and 0.5ul of cDNA was used for each qPCR reaction, utilizing the KAPA SYBR $®$ FAST Universal $2 X$ qPCR Master Mix. qPCR primers are listed within the Supplemental Data.

Lentivirus production. Lentiviral particles were generated by transfecting 293T cells with the pSB700 sgRNAexpression plasmid (with cerulean reporter) and the psPAX2 and pMD2.G (Addgene \#12260 and \#12259) packaging vectors at a ratio of $4: 3: 1$, respectively. Viral supernatants were collected 48-72h following transfection and concentrated using the PEG Virus Precipitation Kit (BioVision) according to the manufacturer's protocol.

iPSC culture and dCas9-AD cell line generation. PGP1 iPS cells were obtained from the Coriell Institute Biorepository (GM23338) and maintained on matrigel (Corning) coated tissue culture plates in mTeSR Basal medium (Stemcell technologies). To generate stable iPS Cas9-AD expressing cell lines, approximately $5 \times 10^{\wedge} 5$ cells were nucleofected with 1.5ug of Cas9-AD piggy-bac expression vector and 340ng of transposase vector (System Biosciences) using the Amaxa P3 Primary Cell 4D-Nucleofector X Kit (Lonza), program CB-150. Following electroporation, cells were seeded onto 24-well matrigel-coated plates in the presence of 10uM ROCK inhibitor (R\&D systems) and allowed to recover for two days before expanding to 6 -well plates in the presence of $20 \mathrm{ug} / \mathrm{ml}$ hygromycin to select for a mixed population of Cas9-AD integrant containing cells.

iPSC transduction and neural induction. For neural induction experiments, iPS Cas9-AD cell lines were transduced with lentiviral preparations containing 30 sgRNAs, targeted against NEUROD1 or NGN2, one day after seeding onto matrigel coated plates. Transduced cells were expanded and then sorted for the top $15 \%$ of cerulean positive cells (pSB700 gRNA expression). Once sorted, sgRNA containing Cas9-AD iPS cell lines were obtained and seeded in triplicates onto matrigel coated 24-well plates with $\mathrm{mTeSR}+10 \mathrm{uM}$ ROCK inhibitor, either in the presence or absence of $1 \mathrm{ug} / \mathrm{ml}$ of doxycycline. Fresh mTeSR medium + or - doxycycline was added every day for 4 days, at which cells were analyzed by immunofluorescence and harvested for qRT-PCR analysis.

Immunostaining of Cas9 iNeurons. All steps for staining were performed at room temperature. Samples were washed once with PBS then fixed with $10 \%$ formalin (Electron Microscopy Sciences) for $20 \mathrm{~min}$ followed by permeabilization with $0.2 \%$ Triton X-100/PBS for 15 min. Samples were then blocked with $8 \%$ BSA for 30 min followed by staining with primary antibodies diluted into $4 \%$ BSA. Staining was performed for either $3 \mathrm{~h}$ with anti-Beta III eFluor 660 conjugate (eBioscience) or $1 \mathrm{~h}$ with anti-neurofilament 200 (Sigma), both at a 1:500 dilution. Samples were then washed 3 times, 5 minutes each, with $0.1 \%$ tween/PBS, followed by one wash with PBS. For neurofilament 200 staining a secondary donkey anti-rabbit Alexa Fluor 647 (Life Sciences) antibody was added at a 1:1000 dilution in $4 \%$ BSA for $1 \mathrm{~h}$. Samples were again washed as previously mentioned then stained with nucBlue [Hoechst 33342] (Life Sciences) for $5 \mathrm{~min}$.

Image acquisition and analysis of Cas9 iNeurons. 24well plates stained for NucBlue and neuronal markers, were imaged with a 10x objective on a Zeiss Axio Observer Z1 microscope. Zen Blue software (Zeiss) was used to program acquisition of 24 images per well. Total cell (NucBlue) and 
iNeuron (Beta III tubulin or neurofilament 200) counts were quantified for each image using custom Fiji and Matlab scripts and used to determine the \% of iNeurons per well by the formula:

(\# Beta III positive cells / \# nucBlue cells) x 100

Images were post-processed and artificially colored for display in this publication (all adjustments were applied equally across the images).

Statistical analysis. All statistical comparisons are twotailed t-tests calculated using the GraphPad Prism software package (Version 6.0 for Windows. GraphPad Software, San Diego, CA). All sample numbers listed indicate the number of biological replicates employed in each experiment.

\section{ACKNOWLEDGEMENTS}

We thank Kevin Esvelt, Prashant Mali and the rest of the members of the Church and Collins labs for helpful discussions. We thank Thomas C. Ferrante, Susan Byrne and Michael Farrell for technical assistance, Albert Keung for providing zinc-finger reporter constructs, and Joanna Schulak for graphic design. This work was supported by US National Institutes of Health NHGRI grant P50 HG005550, US Department of Energy grant DE-FG02-02ER63445 and the Wyss Institute for Biologically Inspired Engineering. A.C. acknowledges funding by the National Cancer Institute grant 5T32CA009216-34. S.V. acknowledges funding by the National Science Foundation Graduate Research Fellowship Program, the Department of Biological Engineering at MIT, and the Department of Genetics at Harvard Medical School.

\section{AUTHOR CONTRIBUTIONS}

A.C. and J.R.S. conceived of the study. A.C., J.R.S., and S.V. designed and performed experiments and interpreted data. B.P. designed and developed fusion libraries. M.T., D.T-O., C.D.G., D.J.W., performed experiments. N.D. and J.L.B. developed reagents. E.I. performed the iNeuron analysis. S.K. and R.W. designed, tested and analyzed the TALE activator data. J.A. designed a subset of the gRNAs. J.J.C and G.M.C. supervised the study. A.C., J.R.S., S.V. and B.P. wrote the manuscript with support from M.T. and all authors.

\section{COMPETING FINANCIAL INTERESTS}

G.M.C. is a founding member of Editas Medicine, a company that applies genome editing technologies.

\section{REFERENCES}

1. Mali, P. et al. CAS9 transcriptional activators for target specificity screening and paired nickases for cooperative genome engineering. Nat. Biotechnol. 31, 833-838 (2013).

2. Gilbert, L. A. et al. CRISPR-mediated modular RNA-guided regulation of transcription in eukaryotes. Cell 154, 442-451 (2013).

3. Maeder, M. L. et al. CRISPR RNA-guided activation of endogenous human genes. Nat. Methods 10, 977-979 (2013).

4. Cheng, A. W. et al. Multiplexed activation of endogenous genes by CRISPR-on, an RNA-guided transcriptional activator system. Cell Res. 23, 1163-1171 (2013).

5. Perez-Pinera, P. et al. RNA-guided gene activation by CRISPRCas9-based transcription factors. Nat. Methods 10, 973-976 (2013).

6. Garneau, J. E. et al. The CRISPR/Cas bacterial immune system cleaves bacteriophage and plasmid DNA. Nature 468, 67-71 (2010).

7. Gasiunas, G., Barrangou, R., Horvath, P. \& Siksnys, V. Cas9crRNA ribonucleoprotein complex mediates specific DNA cleavage for adaptive immunity in bacteria. Proc. Natl. Acad. Sci. U. S. A. 109, E2579-2586 (2012).

8. Deltcheva, E. et al. CRISPR RNA maturation by trans-encoded small RNA and host factor RNase III. Nature 471, 602-607 (2011).

9. Jinek, M. et al. A programmable dual-RNA-guided DNA endonuclease in adaptive bacterial immunity. Science 337, 816821 (2012).

10. Mojica, F. J. M., Díez-Villaseñor, C., García-Martínez, J. \& Almendros, $\mathrm{C}$. Short motif sequences determine the targets of the prokaryotic CRISPR defence system. Microbiol. Read. Engl. 155, 733-740 (2009).

11. Cho, S. W., Kim, S., Kim, J. M. \& Kim, J.-S. Targeted genome engineering in human cells with the Cas9 RNA-guided endonuclease. Nat. Biotechnol. 31, 230-232 (2013).

12. Cong, L. et al. Multiplex genome engineering using CRISPR/ Cas systems. Science 339, 819-823 (2013).

13. Mali, P. et al. RNA-guided human genome engineering via Cas9. Science 339, 823-826 (2013).

14. Jinek, M. et al. RNA-programmed genome editing in human cells. eLife 2, e00471 (2013).

15. Sapranauskas, R. et al. The Streptococcus thermophilus CRIS$\mathrm{PR} /$ Cas system provides immunity in Escherichia coli. Nucleic Acids Res. 39, 9275-9282 (2011).

16. Qi, L. S. et al. Repurposing CRISPR as an RNA-guided platform for sequence-specific control of gene expression. Cell 152, 1173-1183 (2013).

17. Mali, P., Esvelt, K. M. \& Church, G. M. Cas9 as a versatile tool for engineering biology. Nat. Methods 10, 957-963 (2013).

18. Hsu, P. D., Lander, E. S. \& Zhang, F. Development and Applications of CRISPR-Cas9 for Genome Engineering. Cell 157, 1262-1278 (2014).

19. Gersbach, C. A. \& Perez-Pinera, P. Activating human genes with zinc finger proteins, transcription activator-like effectors and CRISPR/Cas9 for gene therapy and regenerative medicine. Expert Opin. Ther. Targets 18, 835-839 (2014).

20. Gersbach, C. A. Genome engineering: the next genomic revolution. Nat. Methods 11, 1009-1011 (2014). 
21. Poss, Z. C., Ebmeier, C. C. \& Taatjes, D. J. The Mediator complex and transcription regulation. Crit. Rev. Biochem. Mol. Biol. 48, 575-608 (2013).

22. Chen, L.-W. et al. Two phenylalanines in the C-terminus of Epstein-Barr virus Rta protein reciprocally modulate its DNA binding and transactivation function. Virology 386, 448-461 (2009).

23. Spitz, F. \& Furlong, E. E. M. Transcription factors: from enhancer binding to developmental control. Nat. Rev. Genet. 13, 613-626 (2012).

24. Esvelt, K. M. et al. Orthogonal Cas9 proteins for RNA-guided gene regulation and editing. Nat. Methods 10, 1116-1121 (2013).

25. Robinton, D. A. \& Daley, G. Q. The promise of induced pluripotent stem cells in research and therapy. Nature 481, 295-305 (2012).

26. Busskamp, V. et al. Rapid neurogenesis through transcriptional activation in human stem cells. Mol. Syst. Biol. 10, n/an/a (2014).

27. Zhang, Y. et al. Rapid Single-Step Induction of Functional Neurons from Human Pluripotent Stem Cells. Neuron 78, 785-798 (2013).

28. Gingras, M., Champigny, M.-F. \& Berthod, F. Differentiation of human adult skin-derived neuronal precursors into mature neurons. J. Cell. Physiol. 210, 498-506 (2007).

29. Manet, E. et al. The acidic activation domain of the EpsteinBarr virus transcription factor $\mathrm{R}$ interacts in vitro with both TBP and TFIIB and is cell-specifically potentiated by a prolinerich region. Gene Expr. 3, 49-59 (1993).

30. Gerritsen, M. E. et al. CREB-binding protein/p300 are transcriptional coactivators of p65. Proc. Natl. Acad. Sci. 94, 2927-2932 (1997).

31. Tanenbaum, M. E., Gilbert, L. A., Qi, L. S., Weissman, J. S. \& Vale, R. D. A Protein-Tagging System for Signal Amplification in Gene Expression and Fluorescence Imaging. Cell 159, 635 - 646 (2014).

32. Gaspar-Maia, A., Alajem, A., Meshorer, E. \& Ramalho-Santos, M. Open chromatin in pluripotency and reprogramming. Nat. Rev. Mol. Cell Biol. 12, 36-47 (2011).

33. Maeder, M. L. et al. Targeted DNA demethylation and activation of endogenous genes using programmable TALE-TET1 fusion proteins. Nat. Biotechnol. 31, 1137-1142 (2013).

34. Kearns, N. A. et al. Cas9 effector-mediated regulation of transcription and differentiation in human pluripotent stem cells. Dev. Camb. Engl. 141, 219-223 (2014).

35. Yang, X. et al. A public genome-scale lentiviral expression library of human ORFs. Nat. Methods 8, 659-661 (2011).

36. Kornblihtt, A. R. et al. Alternative splicing: a pivotal step between eukaryotic transcription and translation. Nat. Rev. Mol. Cell Biol. 14, 153-165 (2013). 